Overexpression of SENP7 with an expression vector or mock control vector was used to study the influence on MMP-9 expression $48 \mathrm{~h}$ after transfection.

Results SENP7 was strongly enhanced in synovial tissues from patients with RA. More precisely, we found that SENP7 was highly enriched in the sublining of the RA synovium. In addition, we found upregulated SENP7 expression in RASF vs OASF. Co-staining of SENP7 with cell compartment specific markers showed a co-localisation of SENP7 in the centrosome and within the golgi apparatus. Synovial fibroblasts of hTNFtg mice showed an upregulation of SENP7 both at the mRNA and the protein level vs wild-type fibroblasts. Interestingly, MMP-9 expression was enhanced significantly by overexpression of SENP7 in wild-type fibroblasts after $48 \mathrm{~h}$. In comparison with a mock vector, the presence of the SENP7 expression construct enhanced the MMP-9 expression dramatically. Overexpression studies with other SENP proteins did not alter MMP-9 expression, underlining the fact that the effect is SENP7-driven.

Conclusions Taken together, these findings might explain how the unbalanced SUMOylation network in synovial fibroblasts contributes to the aggressive behaviour of these cells. Futhermore, our data concerning the upregulation of MMP-9 by SENP7 suggest that a strong SUMO imbalance might contribute to the matrix-degrading characteristics of RASF.

\title{
A43 SUMO-SPECIFIC PROTEASE 7 (SENP7) REGULATES MATRIX METALLOPROTEINASE-9 EXPRESSION IN SYNOVIAL FIBROBLASTS
}

S Strietholt, M A Peters, S Frank, N Pundt, S Ender, G Kollias, T Pap Institute of Experimental Musculoskeletal Medicine, Germany

10.1136/ard.2010.129593e

Introduction Small ubiquitin-related modifiers (SUMOs) are key players in post-translational protein modifications and regulate various cellular processes such as cell division or apoptosis. SUMOylation is regulated by a family of SUMO specific proteases (SENPs) known to activate SUMO precursors and regulate deSUMOylation processes of various target proteins. Our previous studies showed that enhanced SUMO expression in rheumatoid arthritis (RA) altered their apoptotic behaviour. Based on these findings and the fact that SENP7 is localised on a susceptibility locus for RA, we analysed the role of SENP7 for the stable activation of rheumatoid arthritis synovial fibroblast (RASF).

Methods Specimens of synovial tissues from RA and OA patients were analysed for the expression of SENP7 by immunohistological staining. The subcellular localisation of SENP7 was determined by immunocytochemistry. Furthermore, SENP7 expression in isolated RASF and osteoarthritis synovial fibroblasts (OASF) or in fibroblasts from wild-type and human tumour necrosis factor transgenic (hTNFtg) mice was analysed by western blot analysis and PCR. The expression of murine matrix metalloproteinase 9 (MMP-9) was determined by ELISA in unstimulated compared with stimulated synovial fibroblasts from wild-type mice (100 ng/ml TNF $\alpha, 24 \mathrm{~h}$ ). 